\title{
Electron impact investigation of hybridization schemes in coupled split-ring resonators
}

\author{
Qiuqun Liang ${ }^{1}$, Yuren Wen ${ }^{1}$, Thomas Reindl ${ }^{2}$, Nahid Talebi $^{1}$, Peter A. van Aken ${ }^{1}$
}

1. Max Planck Institute for Intelligent Systems, Stuttgart Center for Electron Microscopy, Stuttgart, Germany

2. Max Planck Institute for Solid State Research, Stuttgart, Germany

Split-ring resonators (SRRs), the meta-atoms of many magnetic metamaterials with negative permeability [1], have been widely applied to bio-sensing devices [2], antennas [3], perfect metamaterial absorbers [4] and filters [5]. The very wide applicability of SRRs in integrated photonic devices is based on the closedloop electric current that this element sustains, which in a dual representation effectively builds up a magnetic dipole moment. The concomitant induction of electric and magnetic moments in SRRs is utilized to realize negative refractive index metamaterials [1]. Besides its application in photonic circuitry, the electromagnetic interactions between the meta-atoms are also fundamentally interesting for investigating the coupling effects between induced moments. An intuitive understanding of the interaction between the plasmons of adjacent nanoparticles can be provided by the plasmon hybridization method [6], utilizing a hydrodynamic approximation for the conduction electrons. The result of such an approximation can be understood as the formation of bonding and anti-bonding final states. This hybridization method has been investigated in the dipolar limit for several simple geometries.

We present a comprehensive experimental and theoretical investigation of the plasmon hybridization of coupled Au split-ring resonators (SRRs) by means of electron energy-loss spectroscopy (EELS), energyfiltered transmission electron microscopy (EFTEM), discrete dipole approximation (DDA), and Comsol Multiphysics calculations. The samples were patterned in a $30 \mathrm{kV}$ electron beam lithography system (Raith eLINE). A $3 \mathrm{~nm}$ thick $\mathrm{Cr}$ adhesion layer and a $30 \mathrm{~nm}$ thick Au metal layer were deposited on a 30 nm thick $\mathrm{Si}_{3} \mathrm{~N}_{4}$ membrane as a support grid. EELS and EFTEM experiments were performed in the Zeiss SESAM microscope.

EELS can directly measure the photonic local density of states projected along the electron trajectory and directly probe the energy splitting in coupled meta-atoms. Additionally, we can selectively excite particular plasmonic modes by focusing the electron beam onto hotspot regions, since the plasmonic modes sustain unique spatial distributions with hotspots localized along the circumference of the metallic nanostructures. Various trajectories of the electron beam have been used to selectively excite different plasmonic modes in SRRs. In addition, different relative orientations of adjacent split rings have been used to study the hybridization of the induced charges and magnetic moments of the coupled SRRs according to the orientation of the induced moments. An in-depth understanding of the fundamental and high-order multipole plasmon coupling effects of the coupled SRRs, which have been not investigated beforehand, will provide significant perception into the design and optimization of magnetic metamaterials with desirable properties as well as their resonant behavior.

We provide a complete scheme of the multimodal distribution of the coupled and single-element splitring resonators, with a precise attention to the hybridization of those modes according to the induced moment. Our study suggests a clear dominance of electric and magnetic dipole moments for even higher-order modes in the far-field radiation spectrum. The calculated radiated spectra from each individual electric and magnetic moments demonstrate that the role of the dipole moments play a key role in the plasmonic hybridization [7]. 
References:

[1] Veselago, Sov. Phys. Usp. 10 (1968) 509

[2] HJ Lee and J G Yook, Appl. Phys. Lett. 92 (2008) 254103

[3] KB Alici, AE Serebryannikov and E Ozbay, J. Electromagn. Wav. Appl. 24 (2010) 1183

[4] Y Cheng et al, Appl. Phys. A 102 (2011) 99

[5] YD Dong, T Yang and T Itoh, Microw. Theory Tech. 57 (2009) 2211

[6] E Prodan et al, Science 302 (2003) 419

[7] QL gratefully acknowledges financial support from the Doctoral Training Program between Max Planck Society and Chinese Academy of Sciences. NT acknowledges the Alexander von Humboldt Foundation for the research fellowship. The research leading to these results has received funding from the European Union Seventh Framework Program [FP/2007-2013] under grant agreement no 312483 (ESTEEM2).

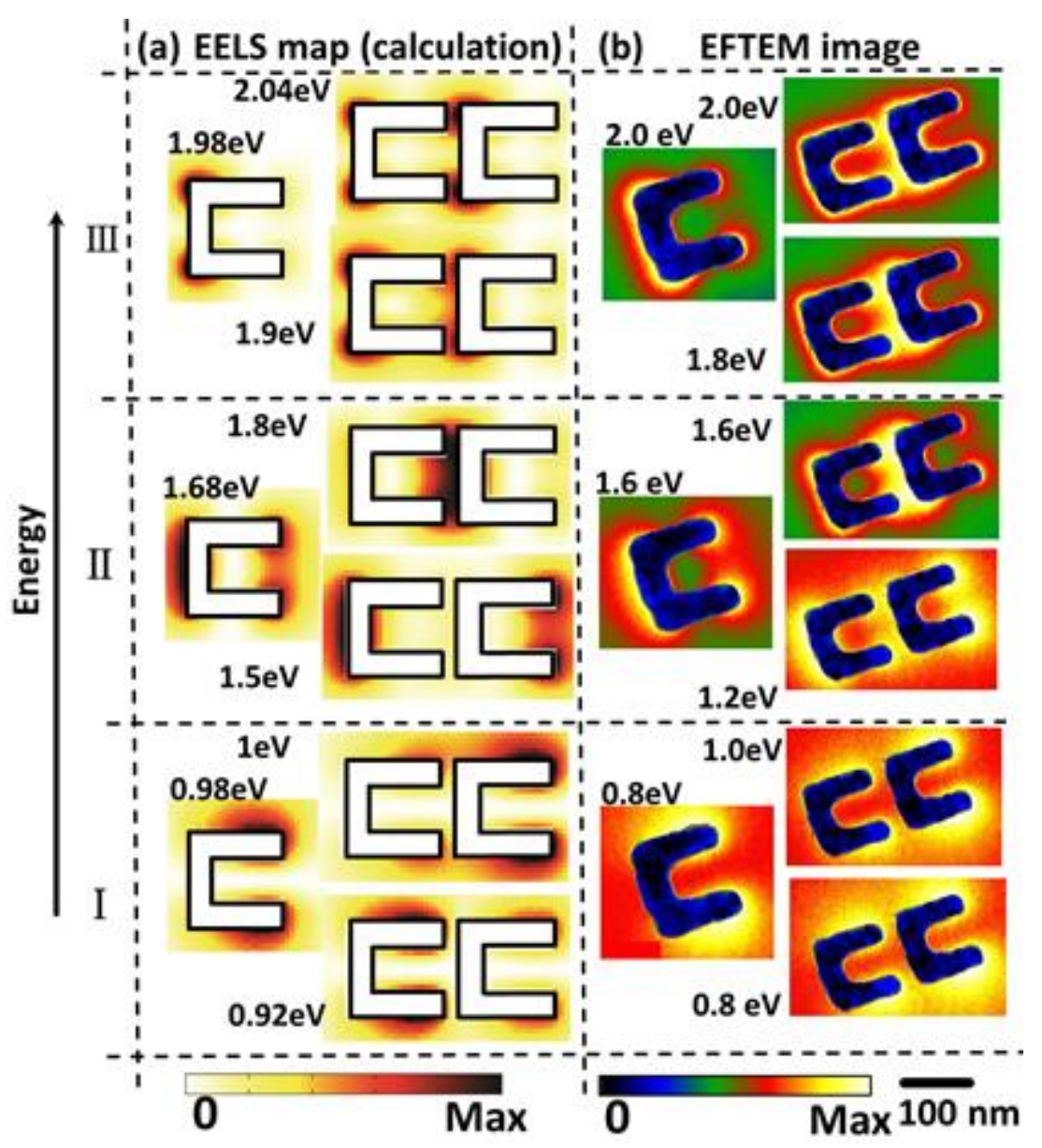

Figure 1. (a) Calculated EELS maps and (b) experimental EFTEM image of the isolated Au SRR structure and a coupled Au SRRs structure at resonance energies of the fundamental, second- and thirdorder plasmonic modes. The parameters of the Au SRRs in the simulations are: thickness $\mathrm{H}=30 \mathrm{~nm}$, length $\mathrm{L}=120 \mathrm{~nm}$, width $\mathrm{W}=30 \mathrm{~nm}$ and gap distance between the left and right SRRs $\mathrm{G}=20 \mathrm{~nm}$. The scale bars of the EFTEM images are $100 \mathrm{~nm}$ and the color codes for the intensities in (a) and (b) are presented accordingly. 